This item was submitted to Loughborough's Research Repository by the author.

Items in Figshare are protected by copyright, with all rights reserved, unless otherwise indicated.

\title{
Quantum downconversion and multipartite entanglement via a mesoscopic superconducting quantum interference device ring
}

PLEASE CITE THE PUBLISHED VERSION

http://dx.doi.org/10.1103/PhysRevB.72.014508

\section{PUBLISHER}

(C) American Physical Society

\section{VERSION}

VoR (Version of Record)

\section{LICENCE}

CC BY-NC-ND 4.0

\section{REPOSITORY RECORD}

Stiffell, P.B., Mark J. Everitt, T.D. Clark, C.J. Harland, and J.F. Ralph. 2019. "Quantum Downconversion and Multipartite Entanglement via a Mesoscopic Superconducting Quantum Interference Device Ring”. figshare. https://hdl.handle.net/2134/12066. 
This item was submitted to Loughborough's Institutional Repository (https://dspace.lboro.ac.uk/) by the author and is made available under the following Creative Commons Licence conditions.

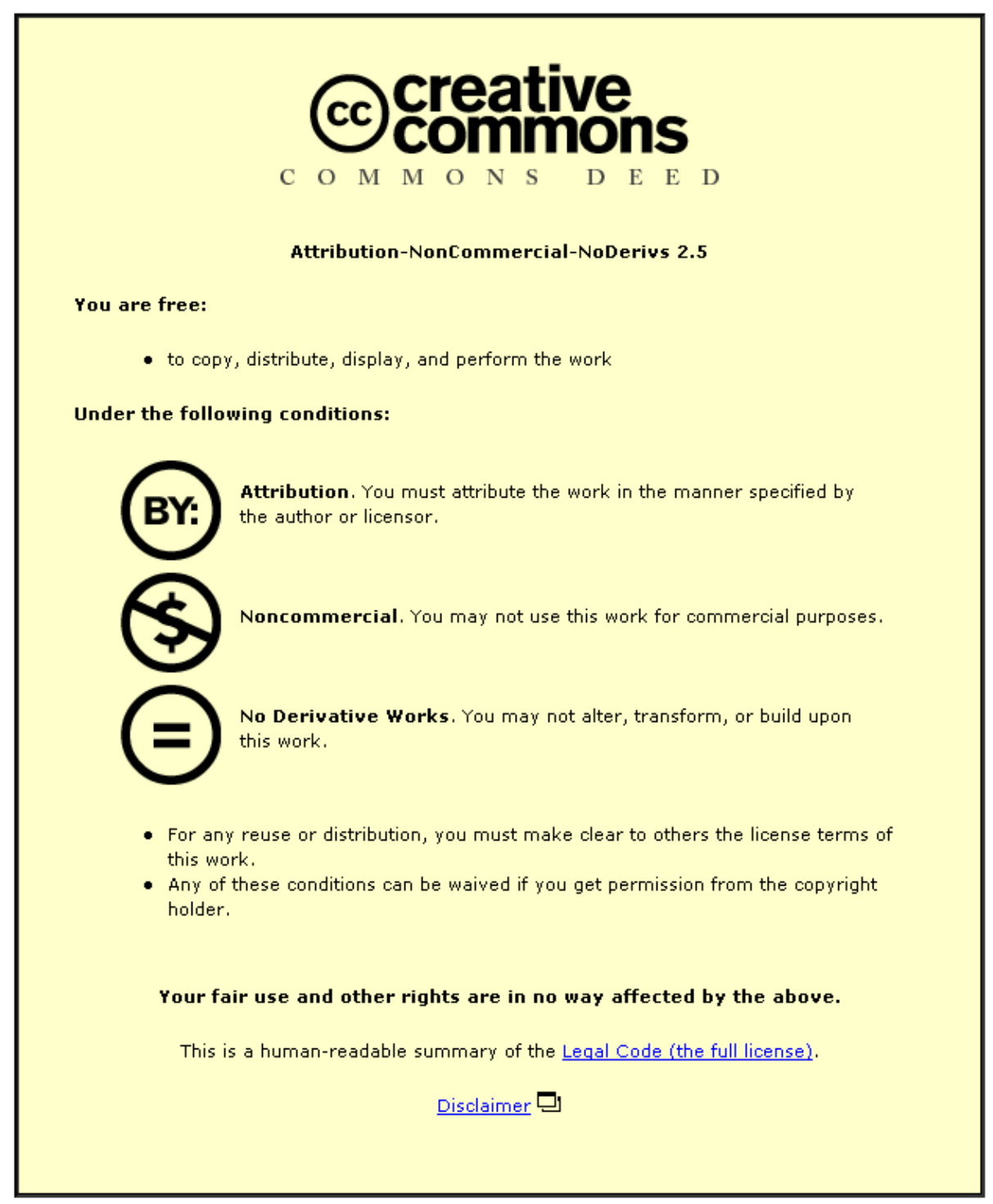

For the full text of this licence, please go to: http://creativecommons.org/licenses/by-nc-nd/2.5/ 


\title{
Quantum downconversion and multipartite entanglement via a mesoscopic superconducting quantum interference device ring
}

\author{
P. B. Stiffell, M. J. Everitt, T. D. Clark,* and C. J. Harland \\ Centre for Physical Electronics and Quantum Technology, School of Engineering, University of Sussex, Brighton, \\ Sussex BN1 9QT, United Kingdom \\ J. F. Ralph \\ Liverpool University, Brownlow Hill, Liverpool L69 3GJ, United Kingdom
}

(Received 22 November 2004; revised manuscript received 30 March 2005; published 6 July 2005)

\begin{abstract}
In this paper we study, by analogy with quantum optics, the superconducting quantum interference device (SQUID) ring mediated quantum mechanical interaction of an input electromagnetic field oscillator mode with two or more output oscillator modes at subintegers of the input frequency. We show that through the nonlinearity of the SQUID ring multiphoton downconversion can take place between the input and output modes with the resultant output photons being created in an entangled state. We also demonstrate that the degree of this entanglement can be adjusted by means of a static magnetic flux which controls the strength of the interaction between these modes via the SQUID ring.
\end{abstract}

DOI: 10.1103/PhysRevB.72.014508

PACS number(s): 74.50. + r, 85.25.Dq, 03.65.-w, 42.50.Dv

\section{INTRODUCTION}

Over the last 5 years there has been a rapidly growing interest in quantum technologies, particularly for the fields of quantum information processing and quantum computing. ${ }^{1,2}$ This has been aided both by the creation of key algorithms ${ }^{3,4}$ and by improvements in experimental technique. A range of approaches, involving different physical disciplines, has been adopted in the pursuit of these technologies but at the basis of each is the concept of using entangled states of quantum objects. ${ }^{1,2}$ The creation of entangled states, and the exploitation of their properties, are therefore seen as fundamental to successful progress in these technologies. In particular, it is clear that there exists a need to develop systems or devices through which the degree of entanglement between quantum objects can be actively controlled. In this paper we show how the intrinsic nonperturbative behavior of a superconducting quantum interference device (SQUID) ring (here, a thick superconducting ring enclosing a Josephson weak link device) can be used to achieve this goal, taking as our specific example entanglement between photon states.

Interest in the use of SQUID rings (and other weak link circuits) as quantum logic devices has been stimulated by recent experiments to probe superposition of states in these systems. ${ }^{5-10}$ Furthermore, other recent experiments have been performed that show coherent dynamics of superconducting flux qubits coupled to individual harmonic oscillators. ${ }^{11}$ Building on this background, and previous theoretical work by us on the coupling of SQUID rings to electromagnetic (em) fields, ${ }^{12}$ we consider the process of photon downconversion via a mesoscopic SQUID ring. Specifically, by analogy with the field of quantum optics, we demonstrate that the ring may be viewed as a nonlinear medium which can downconvert photons from one frequency (input field oscillator mode) to another lower frequency and generate entangled photons (output field oscillator modes), as is often performed in quantum optics experiments. ${ }^{13}$ An analysis of multiple output modes is important since it allows us to understand the way in which several quantum circuits may be entangled - an important issue in quantum technologies-for example, as entanglement registers in quantum computing. We also note that by coupling in a larger number of field modes we change the position (in $\Phi_{x}$ ) of the splittings in the spectrum of the Hamiltonian for the composite system. We consider, therefore, that it is by no means trivial to assume that we can simply extend the results of our previous work. ${ }^{12,14}$ As we have shown by computation, in a three mode system (two em field modes plus one SQUID mode) entanglement can be adjusted through the control of a static bias flux $\Phi_{x}$ applied to the ring. From an experimental viewpoint, in this system downconversion processes are relatively easy to observe ${ }^{15}$ at least for the situation where microwaves (input electromagnetic field $\approx$ in the range of a few to 10 $\mathrm{GHz}$ ) are downconverted to radio frequencies (rf output mode $\approx 1-20 \mathrm{MHz}$ ). ${ }^{15}$ We note that microwave photons are difficult to transport, which could be problematic for some applications in quantum communication. By implication, but not calculated in this work, it should also prove possible to observe upconversion processes involving significant frequency ratios between input and output. However, the ultralow noise electronics required for the output stage in an experiment of this type (say, for inputs at $\mathrm{rf}$ and outputs at microwave frequencies) is much more difficult to engineer than at the much lower radio frequencies. Having observed downconversion in the experiments at Sussex, ${ }^{15}$ we tried to emulate this behavior theoretically. However, such large frequency differences [a few gigahertz $(\mathrm{GHz})$ input, a tetrahertz SQUID ring characteristic oscillator frequency and a few megahertz output] was found to be computationally intractable at this time. Bearing this in mind, and considering the interest currently shown in quantum computing, we chose to demonstrate in this paper that SQUID rings can act as devices which mediate large ratio frequency downconversion that is also computationally tractable. Moreover, for multiple 
output modes we demonstrate that the downconverted photons are entangled together. In our computations we have modeled the field oscillator modes as equivalent resonant $L C$ circuits. Experimentally, this could be realized by utilizing real $L C$ circuits constructed from superconducting material to minimize decoherence. Alternatively we could use a transmission line comprising a series of coupled superconducting LC resonators. ${ }^{15,16}$

From an experimental perspective, it is interesting to note that there has already been some progress made in realizing simple quantum circuits systems relevant to the work presented in this paper. In this regard there are now several examples of coupled superconducting qubits (here the SQUID ring is, to all intents and purposes, a flux qubit). These include fixed coupling flux qubits, ${ }^{17}$ current biased Josephson-junction qubits that are either coupled capacitively, ${ }^{18}$ or via a connecting superconducting loop. ${ }^{19}$ Recently, there has even been an example of tripartite quantum entanglement in macroscopic superconducting circuits. ${ }^{20}$ Moreover, an application that relates very strongly to the circuit systems presented in this work utilized a SQUID ring to mimic a simple harmonic oscillator. This device was then coupled, quantum coherently, to a superconducting flux qubit. ${ }^{11}$ However, although the physics of all these systems is similar, in this work we have chosen to consider a superconducting circuit coupled to quantum electrodynamic degrees of freedom, i.e., photons in a cavity. Recently it has been shown that a superconducting qubit can be quantum coherently coupled to an on-chip cavity. ${ }^{16}$ Furthermore, we note that superconducting devices, which can be coupled to these and more exotic systems, such as $\mathrm{GHz}$ nanomechanical resonators, ${ }^{21}$ are not only easy to fabricate, but are adaptable to a wide variety of applications.

In this work we first investigate, from a full quantum mechanical viewpoint, the simplest downconversion and entanglement process, involving one input photon at a given frequency and two output photons at half this input frequency. To the limits of the computational power available to us, we extend our theoretical investigation of such downconversion processes to that of generating four output photons, each at a quarter of the frequency of the input photon (i.e., a four photon downconversion). Within the Adami-Cerf criterion $^{22-24}$ we show that entanglement exists between any one of these output oscillator modes and the rest of the system (i.e., SQUID ring and input oscillator mode). This implies that all the output modes will be entangled with each other, as well as to the rest of the coupled system. While this multipartite entanglement may not be as strong as some bipartite entanglements, such as Bell states, it is a key component in certain requirements in quantum computation, such as entanglement registers, where a large number of qubits are entangled together to generate a total entangled state for the process of computation. It is this idea that is responsible for the (as yet in principle) power of quantum computing over modern day, classical computers. Also, the question of characterizing entanglement in multipartite quantum systems is still very much an open one. While each measure agrees as to whether the state vector of a given system is separable, or maximally entangled, they do not, in general, provide an ordering that can be used to quantify entanglement of more

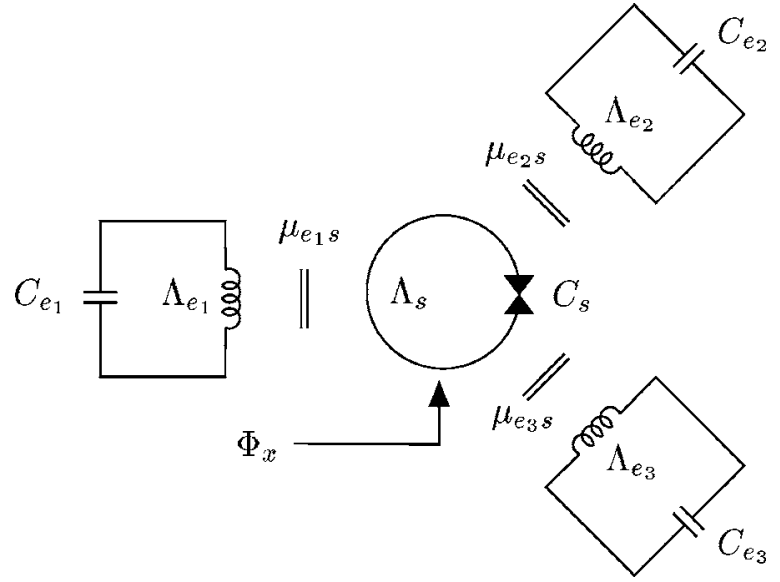

FIG. 1. Schematic of a four mode system comprising three electromagnetic field modes inductively coupled to a mesoscopic SQUID ring.

general states. In this paper we have chosen to use an entanglement measure that gives us an idea of the distribution of the quantum correlations that exist in our example multicomponent systems. However, as we have not produced maximally entangled states it is not possible to quantify absolutely the level of entanglement present. We note that for a simpler system, controlled via a dynamic flux bias, ${ }^{25}$ we have shown that it is possible to create a maximally entangled state between a SQUID ring and an em-field mode. It may be that similar methods could be used in these composite systems to produce such states of maximal entanglement. Unfortunately computations with time dependent Hamiltonians are far harder to solve than the static cases presented in this paper, and are beyond the limit of current computational power.

For the example of the two photon process we consider the effect of dissipation on the coherent evolution of the coupled (entangled) system. By extension, we show by computation that much higher order (i.e., more than our computed four photon case) downconversions may prove possible experimentally. To this effect we provide a further example of downconversion by a factor of 20 between the input em oscillator mode and an output mode. However, both due to limitations in computer power, and a still insufficient understanding of entanglement in many particle (multipartite) quantum systems, we have not proceeded any further in characterizing this high order example.

\section{THEORETICAL MODEL}

In our theoretical treatment of the interaction of quantized em oscillator modes (photons) with a mesoscopic quantum mechanical SQUID ring we model the em field modes as parallel $L C$ resonators, as a simple and convenient way of parameterizing the (physical) cavity modes coupled to the SQUID ring. The scheme we have adopted is shown diagrammatically in Fig. 1 for the simpler, two photon, downconversion process. In this example the input photon frequency is $\omega_{e_{1}} / 2 \pi$ while the two (identical) output 
frequencies are $\omega_{e_{2}} / 2 \pi$ and $\omega_{e_{3}} / 2 \pi$ such that $\omega_{e_{2}}=\omega_{e_{3}}$ $=0.5 \omega_{e_{1}}$. Here, the SQUID ring characteristic oscillator frequency is $\omega_{s} / 2 \pi=1 / 2 \pi \sqrt{\Lambda_{s} C_{s}}$ for a ring inductance and a weak link capacitance of $\Lambda_{s}$ and $C_{s}$, respectively. In our calculations we treat this system fully quantum mechanically by assuming an operating temperature $T$ such that $\hbar \omega_{s}\left(=\hbar / \sqrt{\Lambda_{s} C_{s}}\right), \quad \hbar \omega_{e_{1}}\left(=\hbar / \sqrt{C_{e_{1}} L_{e_{1}}}\right) \quad$ and $\quad \hbar \omega_{e_{2,3}}$ $\left(=\hbar / \sqrt{C_{e_{2,3}} L_{e_{23}}}\right)$ are all much greater than $k_{B} T$ for field mode inductances and capacitances $L_{e_{i}}$ and $C_{e_{i}}$ (where, generally, $i=1,2,3 \ldots)$. In this model system the coupling between the SQUID ring and the oscillator modes is taken to be inductive. As can be seen in Fig. 1, we have chosen to couple these circuits so that all interactions between the field modes are performed via the SQUID ring. In this four mode system (i.e., three em modes plus one SQUID mode) the static flux $\Phi_{x}$ applied to the ring can be used to control the level of interaction between the input em mode and an output em mode of the system. In a specific example in this paper, as an extension of previous work, ${ }^{12}$ we demonstrate that the behavior of coupled SQUID ring-em mode systems is, in general, very strongly dependent on this control flux. Although this aspect of our investigations is an extension of the results of our previous papers, ${ }^{12,14}$ it is important to show that this dependence remains valid for the kind of multicomponent circuits that would appear to be required in the quantum technologies currently being pursued. ${ }^{1,2,5-11,24}$

The Hamiltonian for the coupled system of Fig. 1 is made up of the uncoupled Hamiltonians for each mode together with a set of interaction terms. For each em field mode (both input and output) the Hamiltonian takes the form ${ }^{12}$

$$
H_{e_{i}}=\frac{Q_{e_{i}}^{2}}{2 C_{e_{i}}}+\frac{\Phi_{e_{i}}^{2}}{2 \Lambda_{e_{i}}},
$$

where, again, $i=1,2,3 \ldots$, while for the SQUID ring we use the standard, time independent, lumped component circuit Hamiltonian $^{26,27}$ (the wavelengths of the photon modes being very much larger than the dimensions of the mesoscopic SQUID ring circuit). Specifically

$$
H_{s}=\frac{Q_{s}^{2}}{2 C_{s}}+\frac{\left(\Phi_{s}-\Phi_{x}\right)^{2}}{2 \Lambda_{s}}-\hbar \nu \cos \left(2 \pi \frac{\Phi_{s}}{\Phi_{0}}\right) .
$$

In this description $\Phi_{s}$ (the magnetic flux threading the SQUID ring) and $Q_{s}$ (the electric displacement flux between the electrodes of the weak link) are the conjugate variables for the ring, $\hbar \nu / 2$ is the matrix element for Josephson pair tunneling through the weak link (of critical current $I_{c}=2 e \nu$ ) and $\Phi_{0}=h / 2 e$ is the superconducting flux quantum. From the perspective of the work presented in this paper, the Josephson cosine term in the SQUID ring Hamiltonian (2) allows for nonperturbative behavior to all orders in the coupled system of Fig. 1. In turn, this means that in interactions between a quantum SQUID ring and external em fields, nonperturbative, multiphoton absorption/emission processes tend to dominate within an exchange region. ${ }^{12}$

To illustrate the form of the solutions to the time independent Schrödinger equation for a SQUID ring, using the Hamiltonian (2), we show in Fig. 2(a) the first four eigenen-
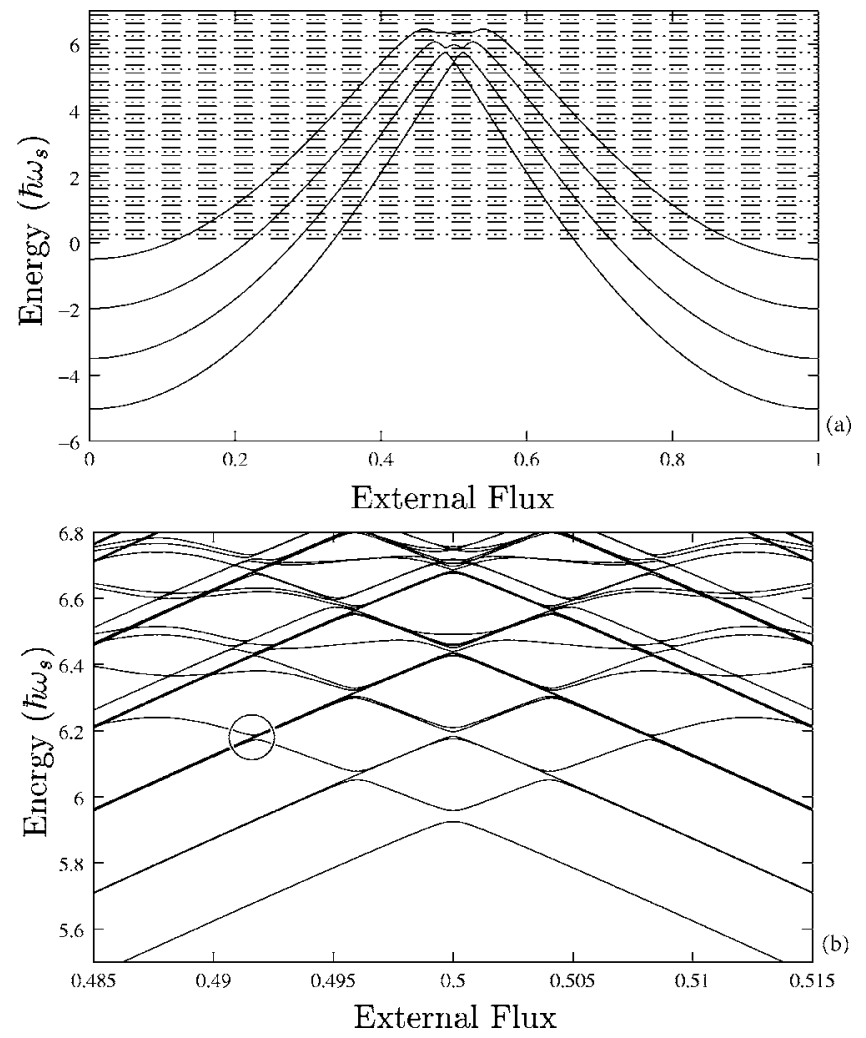

FIG. 2. (a) The eigenenergy spectrum of an uncoupled mesoscopic SQUID ring at $T=0 \mathrm{~K}$ as a function of external bias flux (solid lines) together with the harmonic oscillator spectra for the input mode (dotted) and the two output modes (dashed). (b) The eigenenergy spectrum of the coupled system (Fig. 1) for the four modes coupled together inductively with the circuit parameters given in the text.

ergies $(\kappa=0,1,2,3)$ as a function of external flux $\Phi_{x}$ for a typical mesoscopic quantum SQUID ring with parameters ${ }^{12}$ $C_{s}=5 \times 10^{-15} \mathrm{~F}, \Lambda_{s}=3 \times 10^{-10} \mathrm{H}$ (giving a characteristic SQUID ring oscillator frequency of $130 \mathrm{GHz}$, this is much smaller than the superconducting energy gap in, for example, niobium, that is often used to fabricate SQUID circuits) and $\hbar \nu=0.035 \Phi_{0}^{2} / \Lambda_{s}$ equivalent to a Josephson frequency of 700 $\mathrm{GHz}$, which is not unrealistic when compared to those used by Friedman et al. ${ }^{6}(2 \mathrm{THz})$ and Orlando et al. ${ }^{28}(2.12 \mathrm{THz})$. These frequencies should be sufficient to avoid thermal excitations for any experiments performed at $40 \mathrm{mK}$, a temperature easily obtainable in modern dilution refrigerators. If we assume that the Josephson weak link in the SQUID ring is of the tunnel junction type with, typically, an oxide tunnel barrier of dielectric constant 10, then the junction dimensions will be close to $0.2 \mu \mathrm{m}$ square, which can be fabricated using current lithographic and thin film techniques. Furthermore, with $\hbar \nu=0.035 \Phi_{0}^{2} / \Lambda$, and these dimensions, the corresponding Josephson critical current density is $\approx 4 \mathrm{kA} / \mathrm{cm}^{2}$ which appears suitable for SQUID rings operating in the quantum regime. In addition to the ring eigenenergies, we also show in Fig. 2(a) the harmonic oscillator field mode eigenenergies of $e_{1}\left(\hbar \omega_{e_{1}}=\hbar \omega_{s} / 2\right)$ (dotted) and $e_{2,3}\left(\hbar \omega_{e_{2,3}}\right.$ $\left.=\hbar \omega_{s} / 4\right)$ (dashed). In Fig. 2(b) we show the eigenenergies of the total coupled system. We note that at the level of capaci- 
tance chosen for the SQUID ring the energy splittings where the field mode energies and the ring energies cross are very small. In Fig. 2(b) almost all these splittings are unresolved on this scale but at sufficient resolution all would be discernible.

Provided that we disallow any direct interaction between the field mode oscillators (this being our assumption and a situation which is easy to establish experimentally), the coupling terms are given by

$$
H_{e_{i} s}=\frac{\mu_{e_{i} s}}{\Lambda_{s}}\left(\Phi_{s}-\Phi_{x}\right) \Phi_{e_{i}},
$$

where the $\mu_{e_{i} s}$ are the fractions of magnetic flux coupled inductively between any one of the field mode oscillators and the SQUID ring. The total system Hamiltonian $H_{t}$ for the three em mode system then reads

$$
H_{t}=H_{s}+\sum_{i=1}^{3}\left(H_{e_{i}}-H_{e_{i}}\right) .
$$

We can make a transformation using the unitary operator $T=\exp \left(-i \Phi_{x} Q_{s} / \hbar\right)$ to translate the SQUID Hamiltonian $H_{s}$ into a more convenient form. The Hamiltonian (2) can then be written as ${ }^{12}$

$$
H_{s}^{\prime}=T^{\dagger} H_{s} T=\frac{Q_{s}^{2}}{2 C_{s}}+\frac{\Phi_{s}^{2}}{2 \Lambda_{s}}-\hbar \nu \cos \left(2 \pi \frac{\Phi_{s}+\Phi_{x}}{\Phi_{0}}\right),
$$

where the $\Phi_{0}$-periodic dependence of the (transformed) ring Hamiltonian on $\Phi_{x}$ is explicit. This unitary transformation also modifies the ring-field mode interactions to the form $H_{e_{i} s}^{\prime}=\left(\mu_{e_{i} s} s \Lambda_{s}\right) \Phi_{s} \Phi_{e_{i}}$ so that, following on from (3), the transformed system Hamiltonian (again $\Phi_{0}$-periodic in $\Phi_{x}$ ) can then be written as

$$
H_{t}^{\prime}=H_{s}^{\prime}+\sum_{i=1}^{3}\left(H_{e_{i}}-H_{e_{i}}^{\prime}\right) .
$$

Adopting this Hamiltonian we can solve the eigenproblem $H_{t}^{\prime}\left|\xi_{n}\right\rangle=\Xi_{n}\left|\xi_{n}\right\rangle$ and use the eigenvectors $\left|\xi_{n}\right\rangle$ to construct the evolution operator $U(t)$ for the system ${ }^{12}$ via

$$
U(t)=\sum_{n}\left|\xi_{n}\right\rangle \exp \left(-\frac{i \Xi_{n} t}{\hbar}\right)\left\langle\xi_{n}\right|
$$

The time averaged energy expectation values $\left\langle\left\langle H_{i}\right\rangle\right\rangle$ for the ring and the field modes can then be calculated from the expression

$$
\left\langle\left\langle H_{i}\right\rangle\right\rangle=\lim _{\tau \rightarrow \infty} \int_{0}^{\tau} \operatorname{Tr}\left[\rho_{i}(t) H_{i}\right] d t
$$

where the $\rho_{i}(t)$ are the reduced density operators for the subcomponents $i=e_{1}, e_{2}, e_{3}$ and $s$ for the coupled system ${ }^{29}$ which are obtained from the density operator $\rho(t)=U(t) \rho(0)$ [for an initial density operator $\rho(0)]$ by using $\rho_{i}(t)=\operatorname{Tr}_{j \neq i}\{\rho(t)\}$.
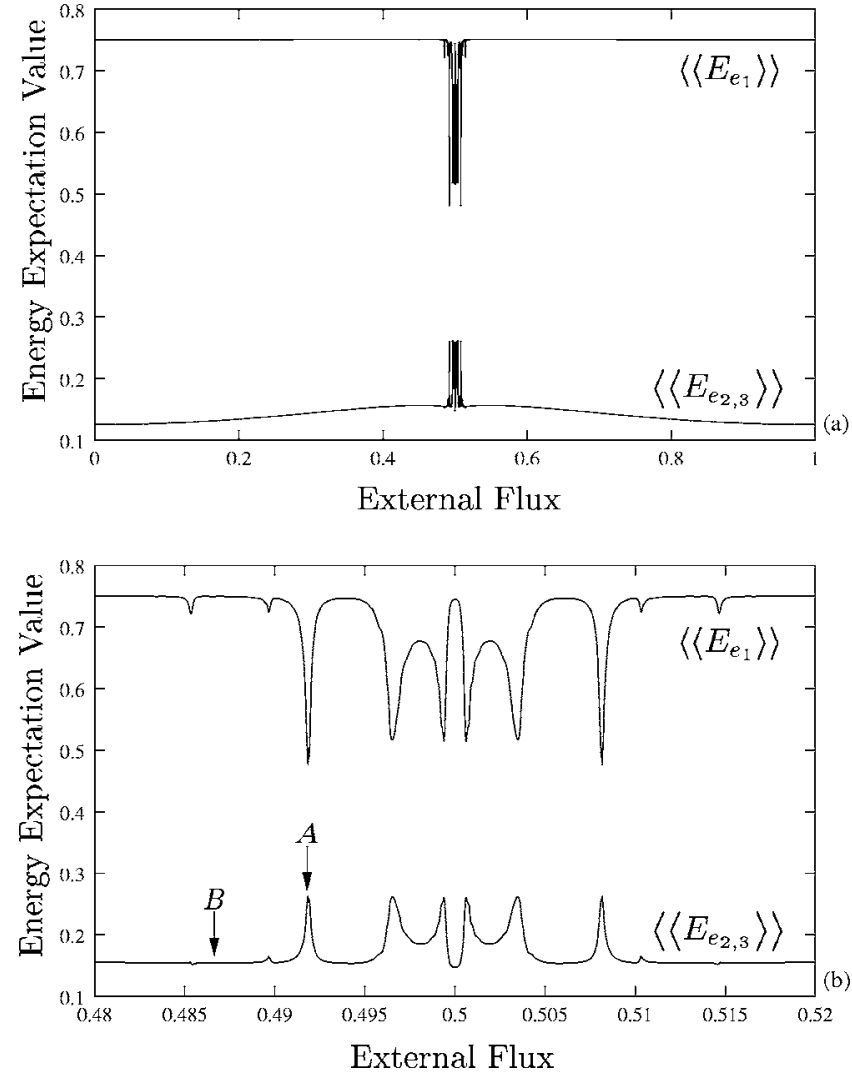

FIG. 3. (a) The time averaged energy expectation values for the electromagnetic input and output modes of the coupled system of Fig. 2 as a function of external bias flux. (b) A zoomed in enlargement of the central section of the results in (a) as a function of bias flux showing a series of energy exchange regions; in the calculations presented subsequently the two bias points $A$ and $B$ have been selected, the former inside an exchange region, the latter outside.

\section{A. Four mode (two photon) downconversion and entanglement}

\section{Without dissipation}

Referring again to the system of Fig. 1, and assuming that it starts in an initial state $|\psi(0)\rangle$, the evolution operator (5) can then be used to generate the system state vector $|\psi(t)\rangle$ $=U(t)|\psi(0)\rangle$ at any subsequent time $t$ (from which $\rho(t)$ can be found via $\rho(t)=|\psi(t)\rangle\langle\psi(t)|)$. As an illustration we now assume that the system, SQUID ring + oscillator modes, starts in the state $|\psi(0)\rangle=|1\rangle_{e_{1}} \otimes|\alpha\rangle_{s} \otimes|0\rangle_{e_{2}} \otimes|0\rangle_{e_{3}}$, where $\alpha$ is the ground state of the ring and the $|n\rangle_{e_{i}}$ are the photon number states for the various field modes of the system.

Starting in this initial state we show in Fig. 3(a) the time averaged energy expectation values of the em field modes ( $e_{1}$ - the input and $e_{2,3}$-the two outputs) of the system as a function of $\Phi_{x}$, where the two outputs $\left(e_{2}\right.$ and $\left.e_{3}\right)$ superimpose exactly, as is to be expected by symmetry. As is apparent, due to the interaction of the SQUID ring with $e_{1}$, and one of the other two (output) modes $e_{2}$ and $e_{3}$, sharp transition regions (or energy exchange regions) ${ }^{12}$ develop in the time averaged energies, discussed by us in a previous publication. ${ }^{12}$ These regions of energy exchange between the ring and the field modes occur over very narrow ranges in 
$\Phi_{x}$, which is clear from our example system in Figs. 3(a) and 3 (b). As we shall demonstrate, the nonperturbative nature of the ring in these exchange regions is sufficient to generate photon downconversion and entanglement between the field modes. In an earlier calculation ${ }^{12}$ we showed that ring-field mode interaction strength is a maximum at the center of the exchange region and very small at its edge. For the calculations presented in this section we have chosen to bias the coupled system at the center of the exchange region, denoted $A$ in Fig. 3(b). The energy exchange region is seen as the energy splitting (divided crossing) circled in Fig. 2(b).

In Figs. 4(a) and 4(b) we show how the number expectation values $\left\langle n_{e_{1}}\right\rangle,\left\langle n_{e_{2}}\right\rangle$, and $\left\langle n_{e_{3}}\right\rangle$ of the three em field modes vary with the dimensionless time $\left(\tau=\omega_{s} t\right)$ for the bias points $A$ and $B$ in Fig. 3(b), where $\Phi_{x}=0.49183 \Phi_{0}$ [at $A$ in Fig. 4(a) ] and $\Phi_{x}=0.487 \Phi_{0}$ [at $B$ in Fig. 4(b)]. Here, we note first that for bias point $A$ there is downconversion of energy from field mode $e_{1}$ to one or other of the two half frequency $\left(\omega_{e_{1}} / 2\right)$ modes $e_{2,3}$ and second that $\left\langle n_{e_{2}}\right\rangle$ and $\left\langle n_{e_{3}}\right\rangle$ vs $\omega_{s} t$ are identical due to the symmetric nature of their coupling to the SQUID ring. In order to verify that the photon occupation of the $e_{2,3}$ modes is due to downconversion from mode $e_{1}$, as stated above, we repeated our calculation at bias point $A$ with the system in the initial state $|\psi(0)\rangle=|0\rangle_{e_{1}} \otimes|\alpha\rangle_{s} \otimes|0\rangle_{e_{2}}$ $\otimes|0\rangle_{e_{3}}$. The time averaged energies $\left\langle n_{e_{2}}\right\rangle$ and $\left\langle n_{e_{3}}\right\rangle$ for this situation are shown in gray in Fig. 4(a). As expected, with $e_{1}$ starting in state $|0\rangle_{e_{1}}$ there was no downconversion. However, we note that there is some occupancy in the output modes due to the ring-mode coupling energy available in the system. It is, of course, possible that higher harmonics of the output field oscillators could be excited, which may not be desirable. In order to investigate such excitations we have included an additional output mode, at the same frequency as the input mode, and recomputed the results of Fig. 4(a). This is shown in Fig. 4(c). Although it is apparent that the level of downconversion is reduced it is, however, still significant. It would appear, therefore, that the presence of unwanted higher harmonics in the output modes is a factor which might need to be reduced. Nevertheless, it does not negate the computations where such processes are not considered.

Concomitant with the two photon down conversion $e_{1}$ $\rightarrow e_{2,3}$ there exists the possibility that the photons in the lower frequency modes $e_{2}$ and $e_{3}$ are strongly correlated or even entangled. In order to characterize the correlations within this system we will employ two entropic quantities. The first of these yields information about the correlations that exist between any two components of a system ${ }^{12,30-33}$ and is defined by

$$
I(A, B)=S_{A}+S_{B}-S_{A B},
$$

where $S_{m}$ is the von Neumann entropy for each subsystem $(m) .{ }^{24}$ We note that for a bipartite closed system this measure is often employed to characterize the entanglement present. However, complications arise when this quantity is used in multipartite or open systems (for more information see Ref. 34). Nevertheless, it is still useful for gaining an appreciation of the correlations that are present between the components of the system in which we are interested. The second en-
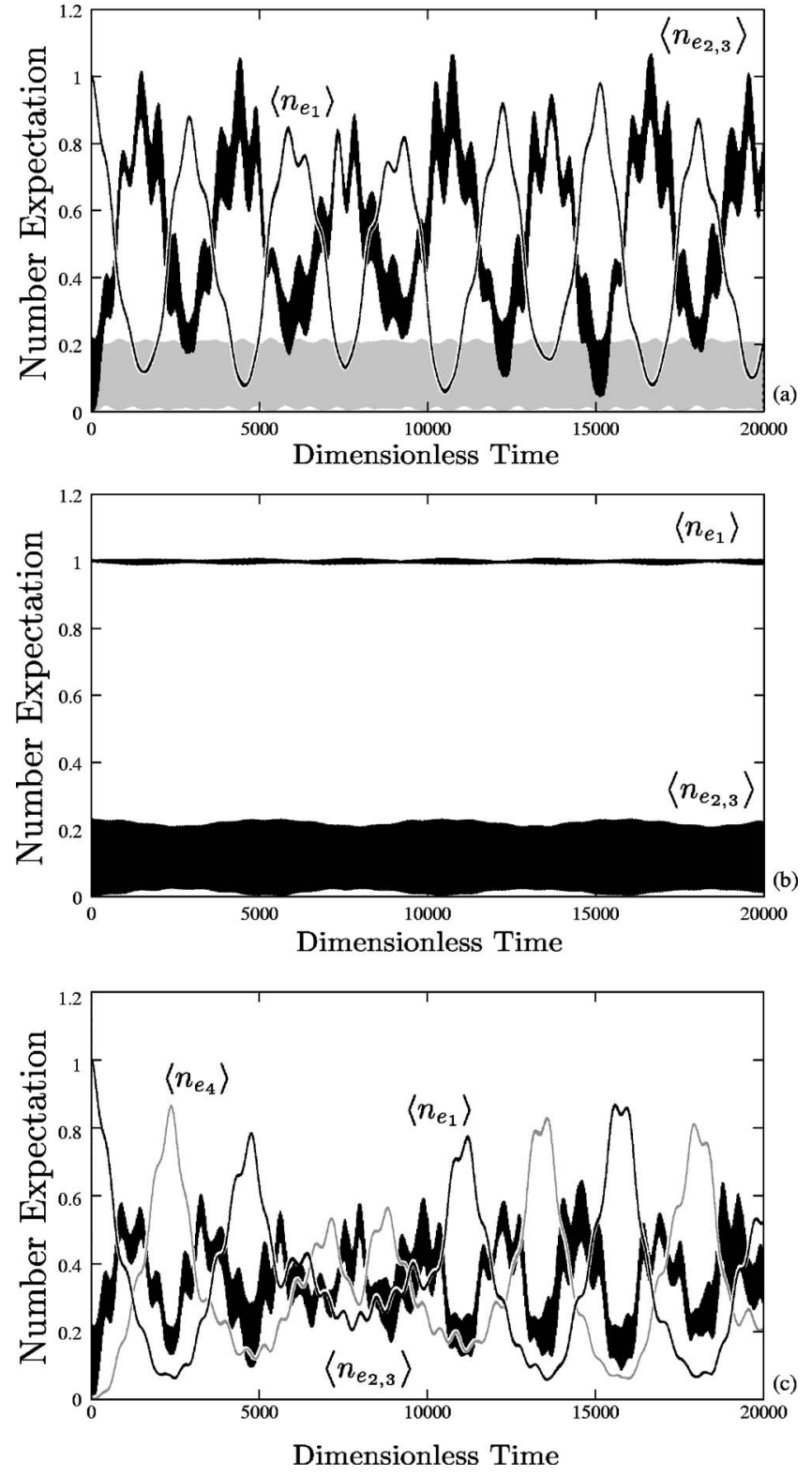

FIG. 4. (a) Number expectation values for the electromagnetic input and output modes of the coupled system of Fig. 2 as a function of dimensionless time, at external flux point $A$ in Fig. 3, starting with a photon occupancy of 1 in the input mode; also shown in gray is the output mode number expectation values for the zero photon occupancy in the input mode. (b) The same results as (a) above calculated at the external bias flux point $B$ in Fig. 3. (c) A repeat of the calculation of (a) with the inclusion of a third output mode $e_{4}$, with its number occupancy shown in gray, at the same frequency as our input mode.

tropic quantity we shall use is the entanglement measure introduced by Adami and Cerf, ${ }^{22}$ which for a subsystem $A$ of the total system $T$ is given by

$$
E(A)=S_{T}-S_{A} .
$$

This measure has the important property that if it is negative definite then $A$ is quantum correlated (i.e., entangled) with at least some of the rest of the system. 

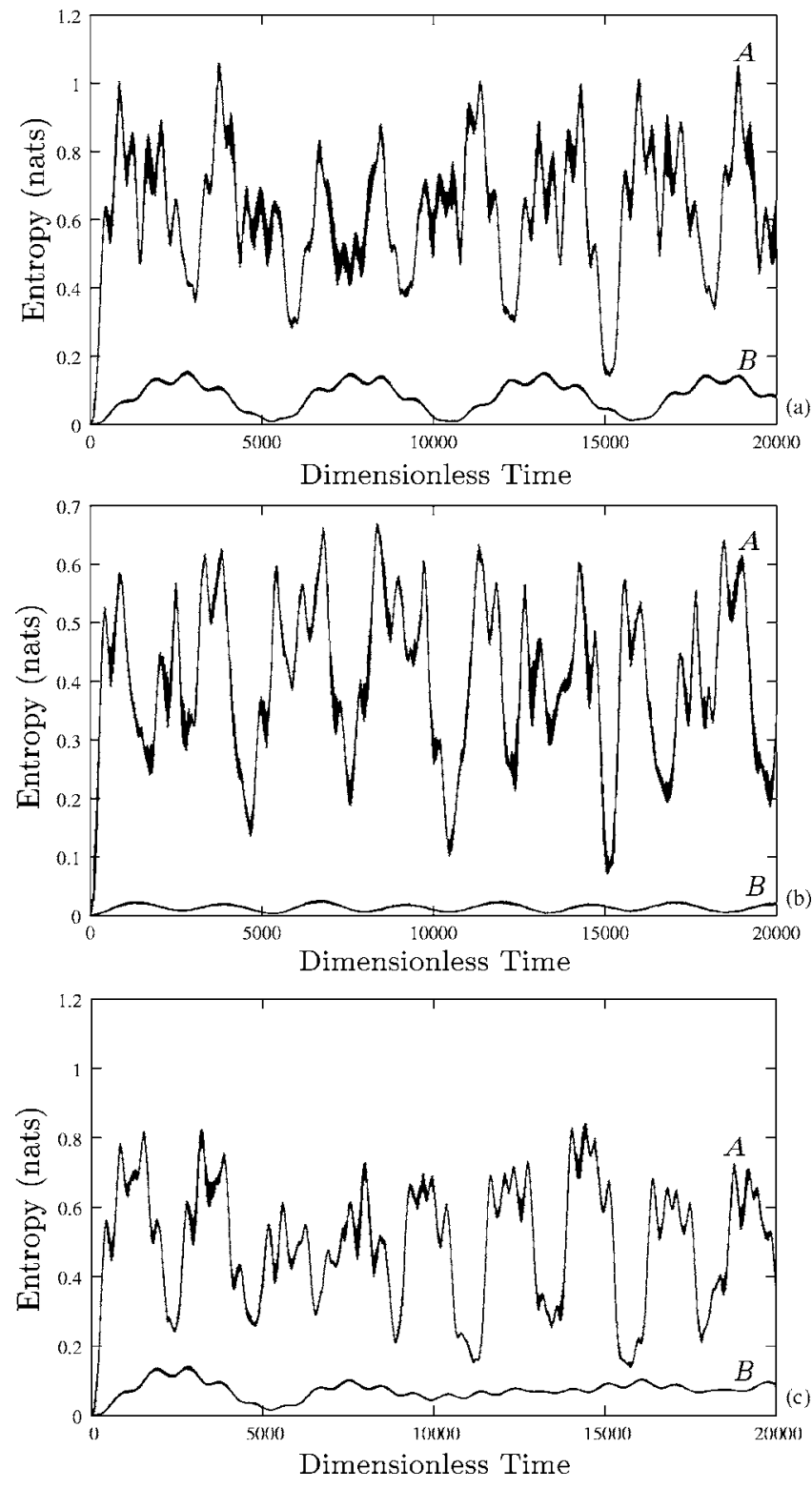

FIG. 5. (a) The entanglement entropy as function of dimensionless time between the two electromagnetic output modes of the system of Fig. 2 calculated at the bias flux points $A$ and $B$ in Fig. 3(b). (b) The entanglement entropy between the input mode and one of the output modes as a function of dimensionless time, again calculated at the two bias points $A$ and $B$ in Fig. 3. (c) A repeat of the calculation of (a) modified by the inclusion of a third output mode at the same frequency as our input mode.

For this particular example we are interested in the correlations that exist between the two output modes, $e_{2}$ and $e_{3}$. The computed entropy, $I\left(e_{2}, e_{3}\right)=S_{e_{2}}+S_{e_{3}}-S_{e_{2}, e_{3}}$, is shown in Fig. 5(a) as a function of dimensionless time $\omega_{s} t$ for the bias points $A$ and $B$ in Fig. 3(b). In addition, in Fig. 5(b) we show the individual, and identical, entropies of the two (receiver) modes $e_{2}$ and $e_{3}$ with the initial em field mode $e_{1}$, i.e., $I\left(e_{1}, e_{i}\right)=S_{e_{1}}+S_{e_{i}}-S_{e_{1}, e_{i}} . i=2$ or 3 for the same two bias points $A$ and $B$. Figure 5(c) shows a repeat of the calculation of Fig. 5(a) having included an additional output mode at the same frequency as the input mode, as in Fig. 4(c). Again, the inclusion of this extra output mode has modified the results. However, the two low frequency output modes $\left(e_{2}\right.$ and $\left.e_{3}\right)$ are still significantly entangled with each other at the appropriate point in external flux, again indicating that the excitation of additional output modes reduces, but does not suppress, the entanglement in between the two low frequency output modes $\left(e_{2}\right.$ and $\left.e_{3}\right)$. We note that the use of an actual LC (Refs. 15 and 16) circuit would altogether remove the need to consider higher order harmonics.

In the photon number expectation values of Fig. 4 it is apparent that the decrease in number expectation value of field mode $e_{1}$ is directly linked to its increase in modes $e_{2}$ and $e_{3}$ and that the degree of transfer is highly dependent on the value of $\Phi_{x}$ in the exchange region. Correspondingly, from Fig. 5(a) it is clear that, with the SQUID ring biased into an exchange region, the correlations between the $e_{2}$ and $e_{3}$ modes are greatly affected by the choice of $\Phi_{x}$. Thus, at $B$ the correlations are relatively close to zero at all times while at $A$ it has a maximum of around 1.1. This $\Phi_{x}$-dependent variation in the strength of correlation is also seen in Fig. 5(b) between the $e_{1}$ and $e_{2}$ (or equivalently $e_{3}$ ) receiver mode. Again these correlations between each pair of coupled modes is identical, whether we choose bias point $A$ or $B$. In order to characterize the quantum correlations with this system we show in Fig. 6 the Adami-Cerf entanglement entropies for each mode of the system where, in Fig. 6(a), for bias point $A$ in Fig. 3(b), all four modes are entangled with the rest of the system throughout the evolution while in Fig. 6(b), while at bias point $B$, it is clear that the level of entanglement is much less. It is apparent in these figures that both the von Neumann and Adami-Cerf approaches indicate that the level of entanglement of the modes in the total system can be controlled by adjusting the external bias flux on the SQUID ring. The control over the entanglement which is manifest in Fig. 6 is to be compared to the case in quantum optics. Here, the optical media used are typically weakly (polynomially) nonlinear as opposed to the extremely strong nonlinear (nonperturbative) properties of the SQUID ring in an exchange region. Furthermore, these media generally couple weakly to em fields which does not have to be the case for SQUID rings. Most importantly, the strength of the ring-field interaction (with the resulting two photon downconversion and entanglement) in an exchange region can be varied by adjusting the bias flux on the ring. To the best of our knowledge, such control is very difficult to achieve with optical media.

\section{With dissipation}

As has been discussed at great length in the literature ${ }^{35}$ coupling of a quantum object to classical dissipative environments leads to decoherence over some characteristic time period. Clearly this is of great importance for any realistic discussion of entanglement in multipartite quantum systems. In order to model the effects of dissipation we have adopted the standard approach, very often used in the field of quantum optics. In this model the components of the system are coupled to decohering thermal baths. The master equation for the evolution of the density of the multicomponent systems then has the form ${ }^{35}$ 

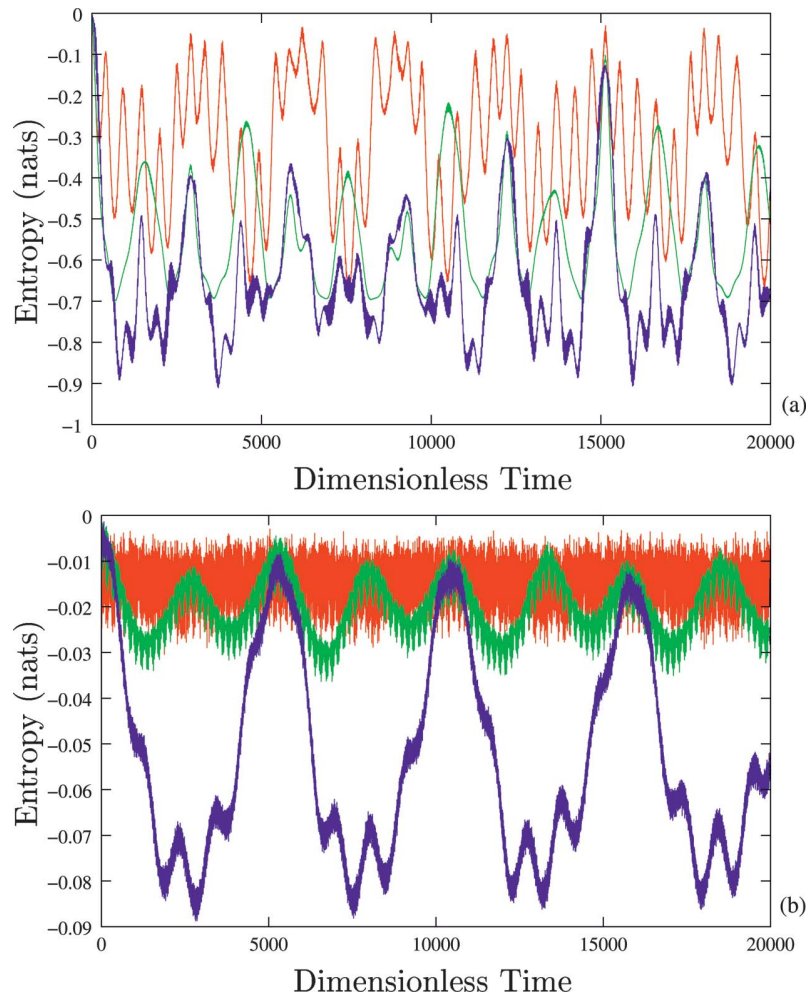

FIG. 6. (Color) (a) The Adami-Cerf entanglement entropies for the coupled system of Fig. 2 as a function of dimensionless time calculated at point $A$ in Fig. 3, where the SQUID ring results are in orange, the input electromagnetic mode results are in green, and those for the two output modes are in purple. (b) The same calculation as above in (a) but now at the external bias flux point $B$ in Fig. 3.

$$
\begin{aligned}
\frac{\partial \rho}{\partial t}= & -\frac{i}{\hbar}\left[H_{t}^{\prime}, \rho\right]+\sum_{\mathrm{sc}} \frac{\gamma_{\mathrm{sc}}}{2 \hbar}\left(M_{\mathrm{sc}}+1\right)\left(2 a_{\mathrm{sc}} \rho a_{\mathrm{sc}}^{\dagger}-a_{\mathrm{sc}}^{\dagger} a_{\mathrm{sc}} \rho\right. \\
& \left.-\rho a_{\mathrm{sc}}^{\dagger} a_{\mathrm{sc}}\right)+\frac{\gamma_{\mathrm{sc}}}{2 \hbar} M_{\mathrm{sc}}\left(2 a_{\mathrm{sc}}^{\dagger} \rho a_{\mathrm{sc}}-a_{\mathrm{sc}} a_{\mathrm{sc}}^{\dagger} \rho-\rho a_{\mathrm{sc}} a_{\mathrm{sc}}^{\dagger}\right),
\end{aligned}
$$

where $M_{\mathrm{sc}}$ is related to the temperature $T=4.2 \mathrm{~K}$, and fre-

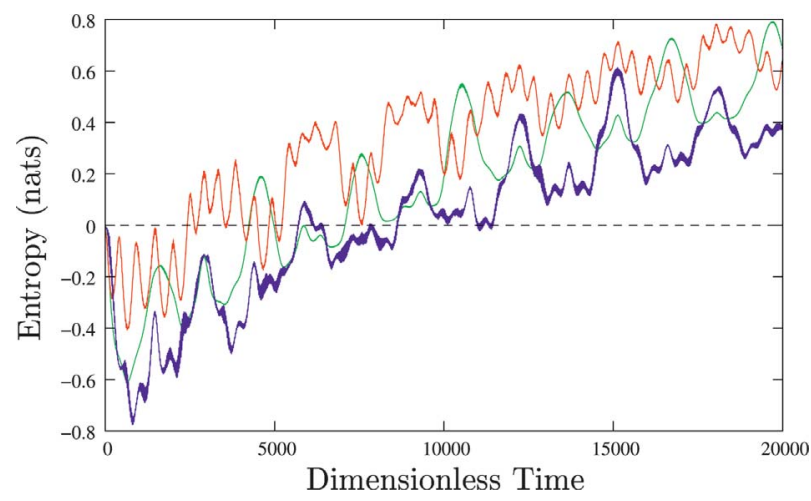

FIG. 7. (Color) Repeat of the calculation in Fig. 6(a) but now with dissipation introduced for a $\gamma=10^{-5} \omega_{s}$ with the color coding green (input mode), purple (output mode), and orange (SQUID ring).

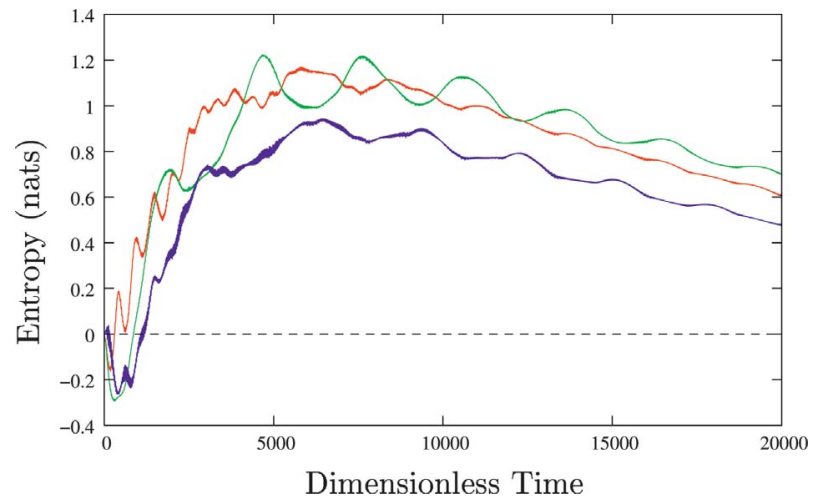

FIG. 8. (Color) Repeat of the calculation in Fig. 6(a) but now with dissipation introduced for a $\gamma=10^{-4} \omega_{s}$ with the color coding green (input mode), purple (output mode), and orange (SQUID ring).

quency $\omega_{\mathrm{bsc}}$, of each decohering bath for each subcomponent (sc) of the system via $M_{\mathrm{sc}}=\left[\exp \left(\hbar \omega_{\mathrm{bsc}} / k_{B} T\right)-1\right]^{-1}$. In Eq. (9) $\gamma_{\mathrm{sc}}$ is the coupling (i.e., the damping rate) between each of the system subcomponents and its respective bath. The choice to couple each subcomponent to a separate decohering bath is in keeping with the standard decoherence model used for open quantum systems. ${ }^{35}$ There is, of course, the possibility that in certain situations all the elements of this system could couple to a mutual decohering bath. ${ }^{36}$ This is an interesting scenario that would require a detailed analysis beyond the scope of this paper.

Following on from the entanglement entropy versus dimensionless time plots for the four mode system in the absence of dissipation (Fig. 6), we show in Figs. 7 and 8 the subcomponent (Adami-Cerf) entropies for two levels of dissipation, namely $\gamma=10^{-5} \omega_{s}$ and $\gamma=10^{-4} \omega_{s}$. As can be seen, in the less dissipative case shown in Fig. 7 (which would appear to be attainable experimentally) 9,37 entanglement is maintained over a relatively long time period. However, as one would expect, over long enough times any original en-

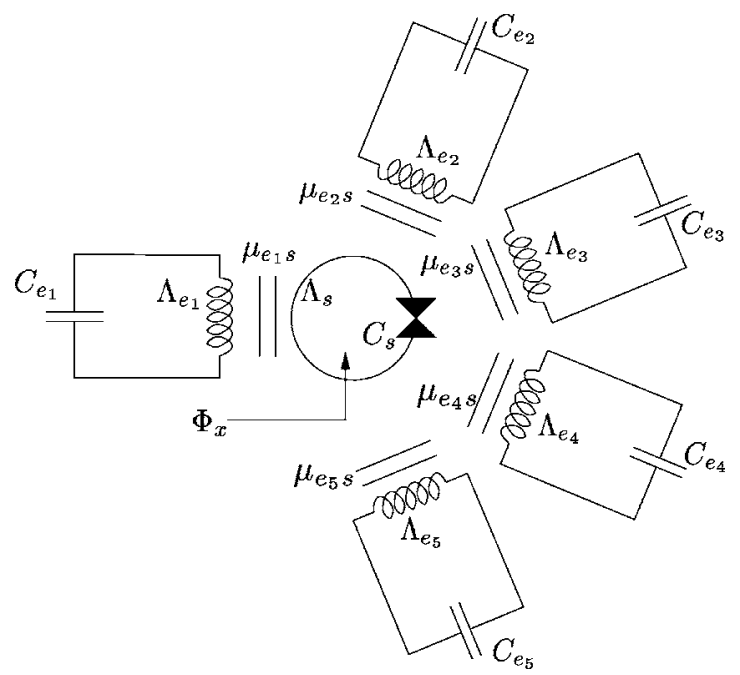

FIG. 9. Schematic for a six mode system with a mesoscopic quantum regime SQUID ring inductively coupled to one input and four output electromagnetic modes. 

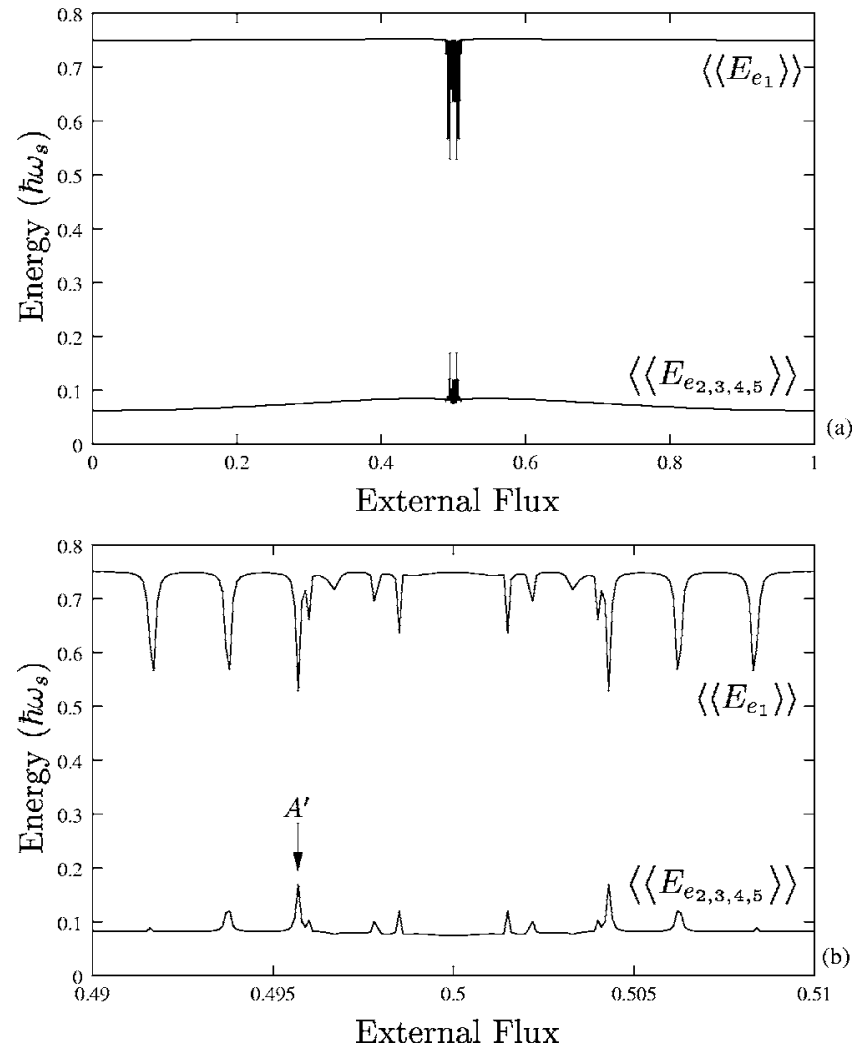

FIG. 10. (a) The time averaged energies at $T=0 \mathrm{~K}$ for the electromagnetic input and output modes of the coupled system of Fig. 9 as a function of external bias flux with the circuit parameters given in the text. (b) A zoomed in enlargement of the central section of the results in (a) as a function of bias flux showing a series of energy exchange regions; in the calculations presented subsequently the bias point $A^{\prime}$ has been selected.

tanglement disappears. Nevertheless, for the case of Fig. 7 it appears that a useful level of entanglement is maintained amongst the output modes over a range of over 5000 in normalized time or $3 \times 10^{-8} \mathrm{~s}$ in real time. This is considerably longer than the time constant corresponding to the $\Lambda_{s} C_{S}$ oscillator frequency of the SQUID ring, i.e., $\left(1 / 2 \pi \sqrt{\Lambda_{s} C_{s}}\right)^{-1}=7 \times 10^{-12} \mathrm{~s}$ for $\Lambda_{s}=3 \times 10^{-10} \mathrm{H}$ and $C_{s}=5$ $\times 10^{-15} \mathrm{~F}$. Thus, provided the level of dissipation can be kept at or below this level it should prove possible to utilize this two photon entanglement in practical situations.

\section{SIX MODE (FOUR PHOTON) DOWNCONVERSION}

The scheme for a six mode system, consisting of one input oscillator mode (frequency $\omega_{e_{1}} / 2 \pi$ ) and four identical output modes, all oscillating at one quarter of the input frequency, is shown diagrammatically in Fig. 9. Using the same procedure as we adopted in the case of the two photon downconversion described above, we have computed the number expectation values against normalized time for the input, the SQUID ring, and the four output modes. These have been computed at the bias flux point $A^{\prime}$ in the center of the exchange region (divided crossing) of Fig. 10. These expectation values are plotted in Fig. 11 for the input mode and the

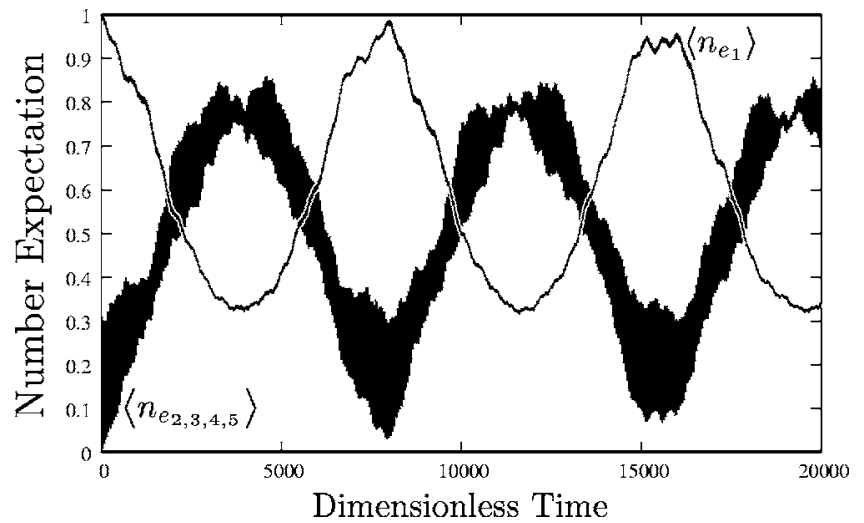

FIG. 11. Number expectation values for the electromagnetic input and output modes of the coupled system of Fig. 9 as a function of dimensionless time, at external flux point $A^{\prime}$ in Fig. 10, starting with a photon occupancy of 1 in the input mode.

four output modes, each of which generates identical results. As can be seen, in the absence of dissipation the sum of the number expectation values for these output modes is reasonably close to 4 , indicating that here the downconversion process via the SQUID ring is quite efficient. We note that the level of efficiency of this downconversion process decreases as the splitting frequency at the divided crossing increases. We suspect that this could be corrected if a sufficiently thorough review of the external flux dependence were to be undertaken. However, this is impractical to perform given the limitations on our current computational power.

The computational power required to generate accurate solutions for this four photon downconversion, and gain a detailed understanding of the correlations that exist between the components of the system, is extremely demanding. As with the two photon downconversion case, but even more so, the computational power at our disposal did not allow us to study the correlations between the four (identical) output modes of the system. What we have been able to do is to take any of the output photons, at a quarter of the frequency of the input photon, and show that each is strongly entangled with the rest of the system. By appealing to the earlier results presented in this paper, it is reasonable to assume that the four output modes are entangled with each other in addition to any entanglement with the input mode and the SQUID ring. The problem of describing, and quantifying, entanglement for systems of more than two quantum particles, is still a matter for serious debate. ${ }^{38,39}$ However, in a qualitative way, the computed results displayed in Fig. 12 suggest a practical route to the entanglement of many identical photon states through the intermediary of a highly nonperturbative SQUID ring medium. Again, with regard to the computational limits we faced, we were not able to introduce dissipation into the many moded system through a master equation of the type (9).

\section{HIGHER ORDER DOWNCONVERSION PROCESSES}

Due to the limitations imposed by our computing power we have not been able to extend our investigations of en- 


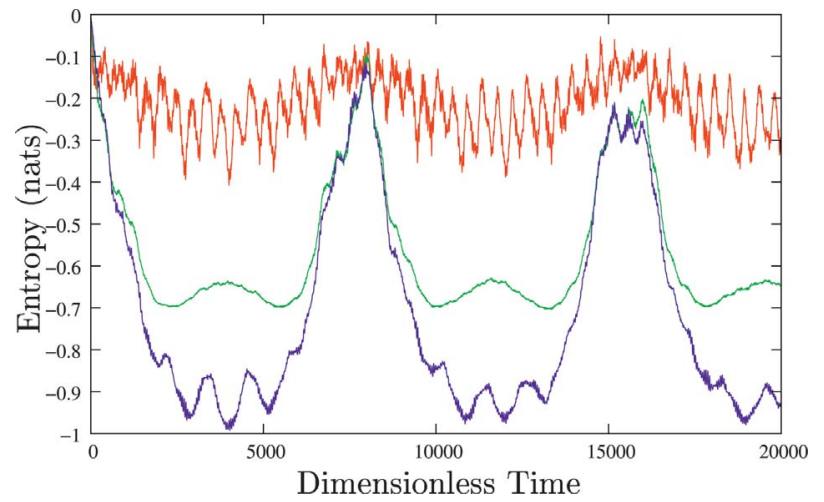

FIG. 12. (Color) The Adami-Cerf entanglement entropies of the for the coupled system of figure as a function of dimensionless time calculated at point $A^{\prime}$ in Fig. 10, where the SQUID ring results are in orange, the input electromagnetic mode results are in green, and those for the two output modes are in purple.

tanglement beyond the six mode (four photon downconversion) range. Nevertheless, following the two and four photon processes we now consider a factor of 20 downconversion process between an input and an output em mode. For this calculation we have used the same SQUID ring parameters as before. However, with this calculation describing a factor of 20 frequency conversion, the exchange regions have been redistributed as shown in Fig. 13. For the calculation presented in this section we have chosen to flux bias the ring at the center of a particular exchange region in Fig. 13, denoted by $A^{\prime \prime}$. At this value of external flux we have been able to demonstrate quantum downconversion by a factor of 20 between the input and an output em oscillator mode. In order to reduce the computational complexity of this calculation, and in contrast to the previous examples, we have taken $\omega_{e_{1}}$ $=\omega_{s}$ and assumed an initial (start) number occupancy in this input mode of $|2\rangle_{e_{1}}$. In the computation we have inductively coupled the SQUID ring to an output oscillator mode at one 20th of the input photon frequency. Setting the input and output coupling strengths at $\mu=0.01$, we should see, for a

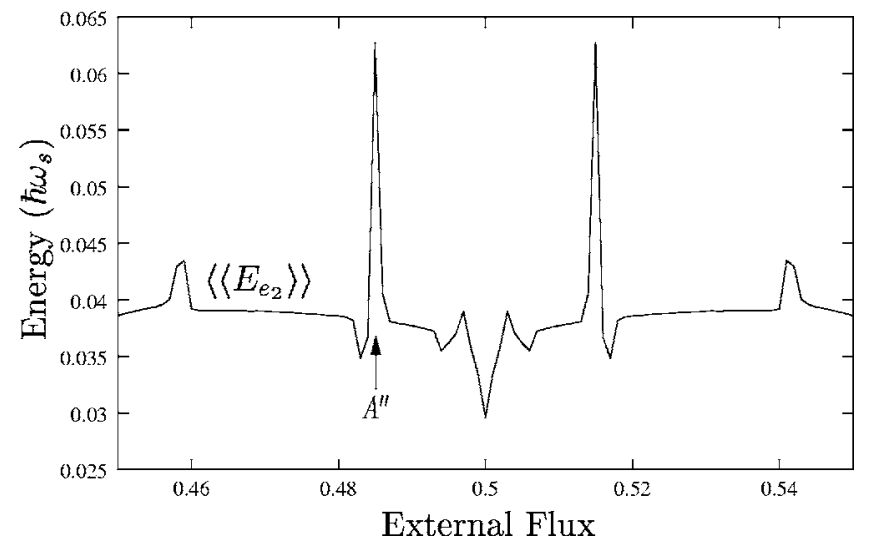

FIG. 13. The $T=0 \mathrm{~K}$ time averaged energy for the output electromagnetic mode of a factor of 20 SQUID ring mediated downconversion process as a function of external bias flux where the bias point $A^{\prime \prime}$ in an energy exchange region is used in subsequent calculations with the circuit parameters given in the text.

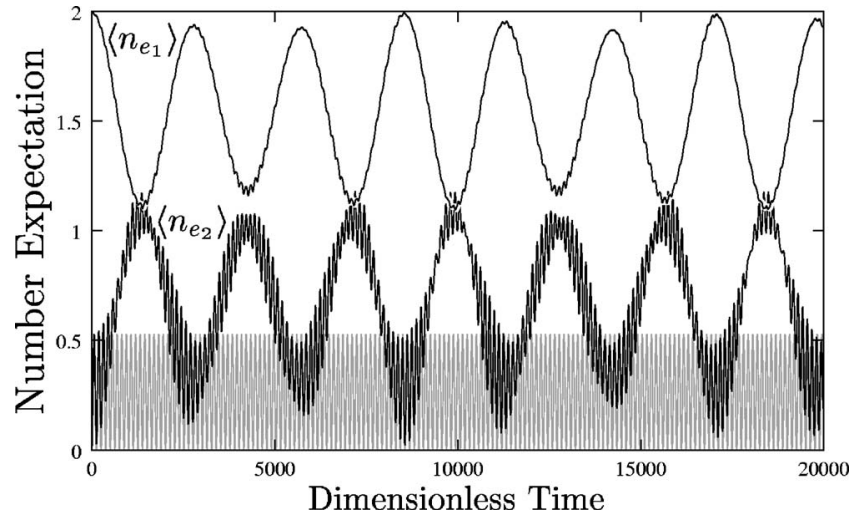

FIG. 14. Number expectation values for the electromagnetic input and output modes of the coupled system of Fig. 13 as a function of dimensionless time, at external flux point $A^{\prime \prime}$ in this figure, starting with a photon occupancy of 2 in the input mode; also shown in gray is the output mode number expectation values for the zero photon occupancy in the input mode.

perfectly efficient downconversion process, that the number expectation value of the output modes reaches a peak of approximately 20 as a function of normalized time. The actual level of occupancy in the output mode is much lower than this potential maximum. However, as discussed earlier, the efficiency of this downconversion process is greatly affected by the value of the external bias flux chosen. The results presented in Fig. 14 are, for the moment, the best that could be obtained within the practical time and computational constraints imposed on us. In Fig. 14 the number expectation value for the output oscillator mode $\left(e_{2}\right)$ is plotted as a function of normalized time. This confirms that, at least from a theoretical viewpoint, high order downconversion (and, by implication, upconversion) processes can occur between input and output em modes through the intermediary of a quantum regime SQUID ring. It should be noted that while the occupancy of the lower frequency output mode here is not very efficient, it is still a nontrivial deviation from zero. It is therefore still valid to look upon this result as evidence that SQUID rings in the quantum regime can mediate high order downconversion, even if, for the moment, at low rates of efficiency. By extension, if the computational power were available to us, it seems reasonable to assume that (i) we could obtain the same result if we were to couple 20 output modes to the SQUID ring and (ii) that there is every possibility that these output modes would be able to entangle with each other. Furthermore, given the extremely nonperturbative nature of a quantum mechanical SQUID ring, we might expect that, with the appropriate experimental arrangements in place, downconversion frequency ratios much higher than 20 might be observed.

\section{CONCLUSIONS}

The flexibility afforded by this $\Phi_{x}$-dependent control (tunability) of the maximum coupling strength, and the temporal form of the entanglements within ring-field mode(s) system, raises interesting possibilities. This control might be of great 
advantage in the preparation of qubits in quantum computing and quantum information processing. ${ }^{1,2}$ Our calculations point to a method for generating a wide range of levels of entanglement between photon states (or, in general, any quantum circuits coupled to the SQUID ring) with only very small adjustments in bias flux required. This we consider to be the important outcome of this work. To give an example, for bias point $A$ in the data of Figs. 5(a) and 5(b) the time evolution shows instances, such as at $\omega_{s} t \approx 10000$, when the entanglement entropy $I\left(e_{2}, e_{3}\right)$ is high and the entanglement entropy $I\left(e_{1}, e_{i}\right), i=2$ or 3 , is low. Even more significantly, in Fig. 7 we see that at dimensionless times such as $\omega_{s} t$ $\approx 5000$ the output modes are the only modes which are unequivocally entangled. This would appear to be of significance in many proposed device applications $s^{1,2,28,40}$ where a way is needed to use a known input to prepare a set of entangled photons and then to disconnect the input, without significantly disrupting the entanglement so produced. We note that in many experimental situations ${ }^{30-33}$ any readjustment of output entanglement requires the development of a separate system setup. Many of the ideas explored in this work were inspired by concepts in quantum optics. In fact, there are strong similarities between a quantum regime SQUID ring and a device frequently employed in quantum optics - the nonlinear optical coupler-this being formed from two or more linearly coupled waveguides where at least one is composed of an optically nonlinear medium. ${ }^{41-45}$ However, there are two important differences between optical couplers and the SQUID ring: (i) optical nonlinear interactions usually have a nonresonant character and (ii) optical nonlinear couplers are difficult to make because of the strict phase and frequency matching conditions that must be obeyed by all involved processes simultaneously. Hence, it may well be that SQUID ring coupled devices, such as those presented here, could lead to a feasible way of realizing (or at least simulating) nonlinears optical couplers.

The manipulation of the different entanglements in the model systems considered in this paper, based on just one SQUID ring, appears to open up the possibility of creating more sophisticated, multi-SQUID ring based, circuit networks where the constituent elements could be entangled and disentangled as required simply by changing bias fluxes. Obviously further work is called for to determine how finely entanglement properties can be manipulated in more complex systems. As a pointer to the use of such complex systems, we have already shown in this work that flux controlled, two photon downconversion $\left(\omega_{1} \rightarrow \omega_{2,3}\right.$ where $\omega_{1}$ $\left.=2 \omega_{2,3}\right)$ and entanglement can occur in a SQUID ring mediated system as can four photon downconversion $\left(e_{1}\right.$ $\left.\rightarrow e_{2,3,4,5}\right)$. Given the cosine nonlinearity of the ring, we would expect the entanglement of even more photon states to be possible although to demonstrate this is beyond the limits of the current computational power available to us.

\section{ACKNOWLEDGMENT}

We would like to express our thanks to the Engineering and Physical Sciences Research Council for the support of this work through its Quantum Circuits Network Initiative.
*Electronic address: t.d.clark@ sussex.ac.uk

${ }^{1}$ Introduction to Quantum Computation and Information, edited by H. K. Lo, S. Popescu, and T. P. Spiller (World Scientific, Hackensack, New Jersey, 1998).

${ }^{2}$ D. Bouwmeester, A. Ekert, and A. Zeilinger, The Physics of Quantum Information (Springer, New York, 2000).

${ }^{3}$ P. Shor, Proceedings of the 35th Annual Symposium on the Foundations of Computer Science (IEEE Computer Society, Los Alamos, CA, 1994), p. 124.

${ }^{4}$ L. K. Grover, Proceedings of the 28th Annual ACM Symposium on Theory of Computing (ACM Press, York, 1996), p. 212.

${ }^{5}$ C. H. van der Wal, A. C. J. ter Haar, F. K. Wilhelm, R. N. Schouten, C. J. P. M. Harmans, T. P. Orlando, S. Lloyd, and J. E. Mooij, Science 290, 773 (2000).

${ }^{6}$ J. R. Friedman, V. Patel, W. Chen, S. K. Tolpygo, and J. E. Lukens, Nature (London) 406, 43 (2000).

${ }^{7}$ Y. Nakamura, C. D. Chen, and J. S. Tsai, Phys. Rev. Lett. 79, 2328 (1997).

${ }^{8}$ Y. Nakamura, Y. A. Pashkin, and J. S. Tsai, Nature (London) 398, 786 (1999).

${ }^{9}$ A. Lupascu, C. J. M. Verwijs, R. N. Schouten, C. J. P. M. Harmans, and J. E. Mooij, Phys. Rev. Lett. 93, 177006 (2004).

${ }^{10}$ T. P. Spiller, Fortschr. Phys. 48, 1075 (2000).

${ }^{11}$ I. Chiorescu, P. Bertet, K. Semba, Y. Nakamura, C. J. P. M. Harmans, and J. E. Mooij, Nature (London) 431, 159 (2004).
${ }^{12}$ M. J. Everitt, T. D. Clark, P. Stiffell, H. Prance, R. J. Prance, A. Vourdas, and J. F. Ralph, Phys. Rev. B 64, 184517 (2001).

${ }^{13}$ M. O. Scully and M. S. Zubairy, Quantum Optics (Cambridge University Press, Cambridge, 1997).

${ }^{14}$ M. J. Everitt, P. Stiffell, T. D. Clark, A. Vourdas, J. F. Ralph, H. Prance, and R. J. Prance, Phys. Rev. B 63, 144530 (2001).

${ }^{15}$ R. Whiteman, T. D. Clark, R. J. Prance, H. Prance, V. Schollman, J. F. Ralph, M J. Everitt, and J. Diggins, J. Mod. Opt. 45, 1175 (1998).

${ }^{16}$ A. Wallraff, D. I. Schuster, A. Blais, L. Frunzio, R. S. Huang, J. Majer, S. Kumar, S. M. Girvin, and R. J. Schoelkopf, Nature (London) 431, 162 (2004).

${ }^{17}$ J. B. Majer, F. G. Paauw, A. C. J. ter Harr, C. J. P. M. Harmans, and J. E. Mooij, Phys. Rev. Lett. 94, 090501 (2005).

${ }^{18}$ A. J. Berkley, H. Xu, R. C. Ramos, M. A. Gubrud, F. W. Strauch, P. R. Johnson, J. R. Anderson, A. J. Dragt, C. J. Lobb, and F. C. Wellstood, Science 300, 1548 (2003).

${ }^{19}$ M. D. Kim and J. Hong, Phys. Rev. B 70, 184525 (2004).

${ }^{20}$ H. Xu, F. W. Strauch, S. K. Dutta, P. R. Johnson, R. C. Ramos, A. J. Berkley, H. Paik, J. R. Anderson, A. J. Dragt, C. J. Lobb, and F. C. Wellstood, Phys. Rev. Lett. 94, 027003 (2005).

${ }^{21}$ M. R. Geller and A. N. Cleland, Phys. Rev. A 71, 032311 (2005).

${ }^{22}$ N. J. Cerf and C. Adami, Phys. Rev. Lett. 79, 5194 (1997).

${ }^{23}$ Feynman and Computation, edited by A. J. G. Hey (Westview Press, Boulder, CO, 1999). 
${ }^{24}$ M. A. Nielsen and I. L. Chuang, Quantum Computation and Quantum Information (Cambridge University Press, Cambridge, 2000).

${ }^{25}$ M. J. Everitt, T. D. Clark, P. Stiffell, H. Prance, R. J. Prance, and J. F. Ralph, quant-ph/0307181 (unpublished).

${ }^{26}$ T. P. Spiller, T. D. Clark, R. J. Prance, and A. Widom, Prog. Low Temp. Phys. 13, 219 (1992).

${ }^{27}$ Y. Srivastava and A. Widom, Phys. Rep. 148, 1 (1987).

${ }^{28}$ T. P. Orlando, J. E. Mooij, L. Tian, C. H. van der Wal, L. Levitov, S. Lloyd, and J. J. Mazo, Phys. Rev. B 60, 15398 (1999).

${ }^{29}$ C. Cohen-Tannoudji, B. Diu, and F. Laloë, Quantum Mechanics (Wiley, New York, 1986), Vol. I, p. 298.

${ }^{30}$ G. Lindbland, Commun. Math. Phys. 33, 305 (1973).

${ }^{31}$ E. H. Leib, Bull. Am. Math. Soc. 81, 1 (1970).

${ }^{32}$ A. Wehrl, Rev. Mod. Phys. 50, 221 (1978).

${ }^{33}$ S. M. Barnett and S. J. D. Phoenix, Phys. Rev. A 44, 535 (1991).

${ }^{34}$ A. J. Scott, Phys. Rev. A 69, 052330 (2004).

${ }^{35}$ U. Weiss, Quantum Dissipative Systems (World Scientific, Singapore, 1999).
${ }^{36}$ T. Yu and J. H. Eberly, Phys. Rev. B 66, 193306 (2002).

${ }^{37}$ J. M. Martinis, S. Nam, J. Aumentado, and C. Urbina, Phys. Rev. Lett. 89, 117901 (2002).

${ }^{38}$ V. M. Kendon, K. Nemoto, and W. J. Munro, J. Mod. Opt. 49, 1709 (2002).

${ }^{39}$ P. Rungta, W. J. Munro, K. Nemoto, P. Deuar, G. J. Milburn, and C. M. Caves, quant-ph/0001075 (2000).

${ }^{40}$ Y. Makhlin, G. Schon, and A. Shnirman, Nature (London) 398, 305 (1999).

${ }^{41}$ D. Mogilevtsev, N. Korolkova, and J. Perina, J. Mod. Opt. 44, 1293 (1997).

${ }^{42}$ J. Rehacek, L. Mista, J. Fiurasek, and J. Perina, Phys. Rev. A 65, 043815 (2002).

${ }^{43}$ J. Fiurasek and J. Perina, Phys. Rev. A 62, 033808 (2000).

${ }^{44}$ J. A. Armstrong, N. Bloembergen, J. Ducuing, and P. S. Pershan, Phys. Rev. 127, 1918 (1962).

${ }^{45}$ C. Silberhorn, P. K. Lam, O. Weiss, F. Konig, N. Korolkova, and G. Leuchs, Phys. Rev. Lett. 86, 4267 (2001). 